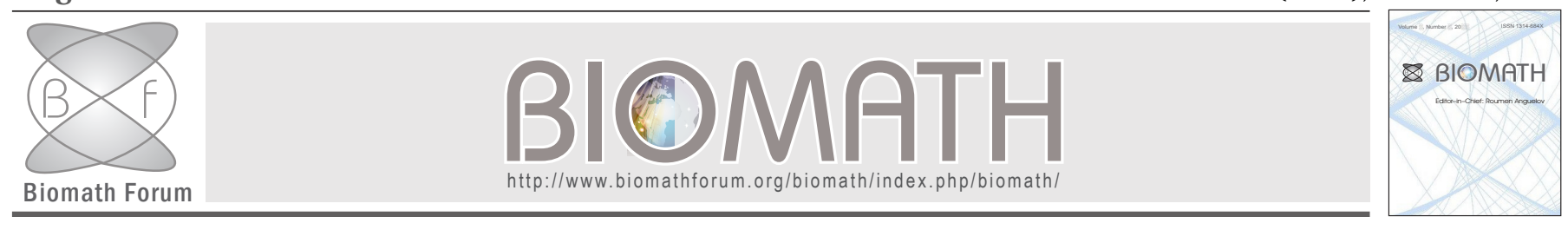

\title{
Viral Dynamic Model of Antiretroviral Therapy Including the Integrase Inhibitor Raltegravir in Patients with HIV-1
}

\author{
Dimitra Bon*, Christoph Stephan ${ }^{\dagger}$, Oliver T. Keppler ${ }^{\ddagger}$ and Eva Herrmann* \\ * Institute of Biostatistic and Mathematical Modeling \\ Department of Medicine, Goethe-University, Frankfurt Main, Germany \\ Emails: bon@med.uni-frankfurt.de, herrmann@med.uni-frankfurt.de \\ $\dagger$ HIV Center
}

Goethe University Hospital and Faculty of Medicine, Frankfurt Main, Germany

Email: Christoph.Stephan@mail.hivcenter.de

‡ Institute of Medical Virology

Goethe University Hospital and Faculty of Medicine, Frankfurt Main, Germany

Email: Oliver.Keppler@kgu.de

Received: 15 July 2012, accepted: 25 September 2012, published: 13 October 2012

\begin{abstract}
Antiviral combination therapies consisting of reverse transcriptase inhibitors, protease inhibitors and an integrase inhibitor, have been developed to suppress HIV below the limit of detection[3]. We introduce a mathematical model for the effect of different combination treatment regimens on the dynamics of HIV RNA and CD4 T-cell counts $[1,7,8,9]$. We will especially focus on modelling the treatment effect of the integrase inhibitorRaltegravir [11]. The model consists of a system of ordinary differential equations and the parameters were chosen or estimated in order to agree with clinical data of a recent clinical trial [13]. All the numerical simulations were calculated with Matlab.
\end{abstract}

Keywords-HIV; mathematical modelling; integrase inhibitor treatment

\section{INTRODUCTION}

Human immunodeficiency virus (HIV) is a member of the retrovirus family. It can cause acquired immunodeficiency syndrome (AIDS) in which the immune system fails, allowing opportunistic infections and cancers to thrive. HIV infection is considered pandemic with approximately 34 million people been infected globally (WHO-UNAIDS 2010)[15].

\section{A. HIV-1 Infection}

In absence of antiretroviral treatment the course of HIV-1 infection consists of three phases.

First phase is the acute phase, it can last several weeks and it is a period of rapid viral replication. The second phase, the chronic phase, can last up to 9-10 years and it is mostly asymptomatic. In this phase an equilibrium is achieved between viral replication and the immune response. The last phase, AIDS, can last a couple of years and the immune system can no longer control the viral replication.[4 (p.6-10)]

\section{B. Replication Cycle and Antiretroviral Treatment (Fig. 1)}

HIV infects cells in the human immune system such as $\mathrm{T}$ cells and in particular the $\mathrm{CD} 4^{+} \mathrm{T}$-cells.

Understanding the HIV replication cycle and how and where the antiretroviral drugs works, helps us to construct our mathematical model. Therefore, we focus on antiviral treatment during the asymptomatic phase of HIV-1 infection.

The process begins when the virus binds to the surface of the CD4 cell. Then RNA enzymes enter the 


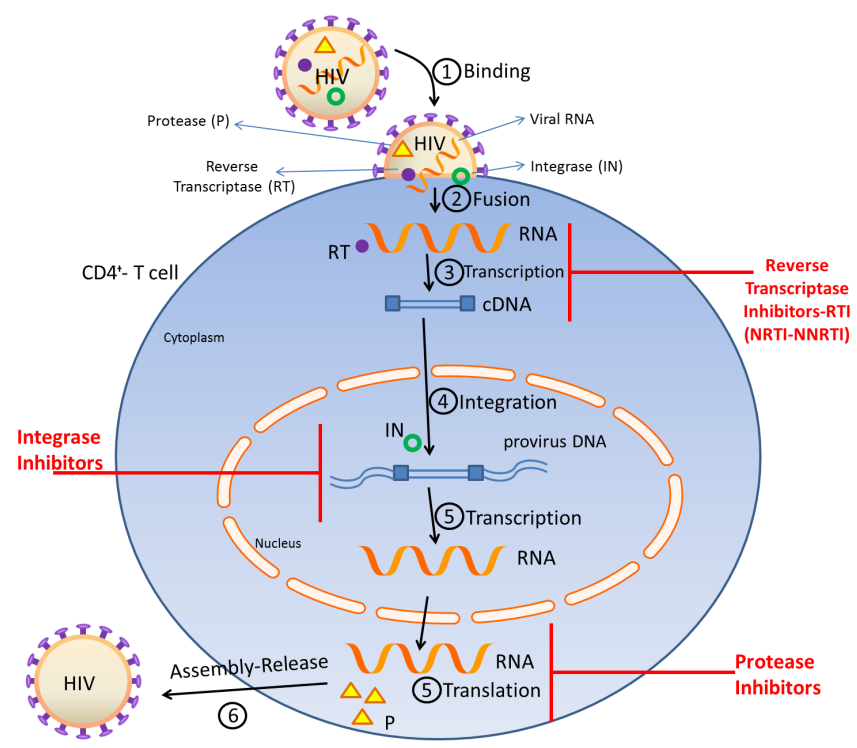

Fig. 1. Replication cycle-Treatment.

cytoplasm. Reverse transcriptase make a DNA copy of the viral RNA and with the help from integrase, the viral DNA integrates into the host DNA. The proviral DNA transcribes into RNA and then RNA is translated into protein. Viral RNA and proteins assembled into viral particles and with the help of protease the new virus buds out.[4 (p.26-33),14]

The management of HIV/AIDS typically includes a combination of antiretroviral drugs and the approach is known as highly active antiretroviral therapy (HAART). The three most important classes of antiretroviral drugs are:

Reverse Transcriptase Inhibitors (RTI) interfere with reverse transcription.

Protease Inhibitors(PI) interfere with the protease enzyme that HIV uses to produce infectious viral particles.

Integrase Inhibitors (INI) block integrase, the enzyme HIV uses to integrate genetic material of the virus into its target host cell. [4 (p.60-94,105-108)]

\section{Modelling the Dynamics of HIV}

We begin with the basic model of untreated chronic viral infection $[7,8,9]$. The model considers three compartments, the uninfected (target) cells $(\mathrm{T})$, the infected cells (I) and the the free virus (V). Target cells are produced at a constant rate $s$ and die at rate $d T$. Free virus infects target cells in order to produce infected cells at rate $k V T$ and dies at rate $c V$. Infected cells die at rate $\delta I$ and produce new virus at rate $N \delta I$.
The equations that describe the basic model are:

$$
\begin{aligned}
& d T / d t=s-k V T-d T \\
& d I / d t=k V T-\delta I \\
& d V / d t=N \delta I-c V
\end{aligned}
$$

where

- $s$ is the source term for target cells

- $d$ is the loss rate of target cells

- $k$ is the infection rate

- $c$ is the loss rate of free virus

- $\delta$ is the loss rate of infected cells

- $N$ is the number of virion production.

Note that we do not model latently infected cell compartments as the models which are discussed in the following mainly aim to model relatively only limited treatment durations.

\section{A. Models of Viral Dynamics under RTI and PI Treat- ment}

Patients infected with HIV-1 were usually treated with RTI's or PI's or a combination of the two. In order to analyse the effect of antiretroviral treatment the model equations become $[8,9]$ :

$$
\begin{aligned}
& d T / d t=s-\left(1-\varepsilon_{2}\right) k V_{I} T-d T \\
& d I / d t=\left(1-\varepsilon_{2}\right) k V_{I} T-\delta I \\
& d V_{I} / d t=\left(1-\varepsilon_{1}\right) N \delta I-c V_{I} \\
& d V_{N I} / d t=\varepsilon_{1} N \delta I-c V_{N I}
\end{aligned}
$$

where

- $\varepsilon_{1}$ and $\varepsilon_{2}$ are the efficacies of PI and RTI ( $0 \leq$ $\left.\varepsilon_{1,2} \leq 1\right)$.

- $V_{I}$ and $V_{N I}$ are the infectious and non-infectious virus, respectively, where $V=V_{I}+V_{N I}$ is the viral load.

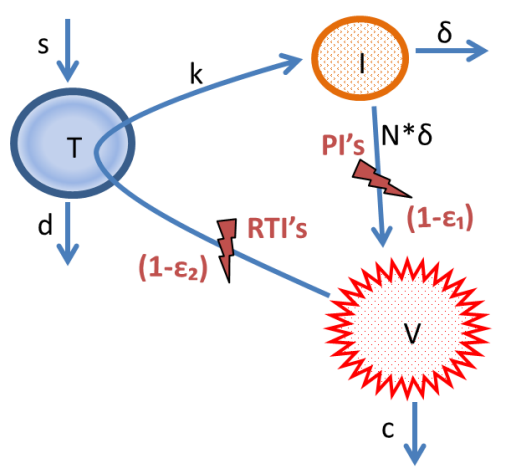

Fig. 2. Model of viral infection under PI and RTI treatment. 


\section{B. Models of Viral Dynamics under Raltegravir Treat- ment}

Since 2007, when Raltegravir was approved by the FDA, we can find in the literature mathematical models that describe the dynamics of HIV-1 under treatment with Raltegravir.

In Sedaghat A. R. et al. [10,11] it is suggested a model with three states, the uninfected, the early stage infection and the late stages infection.

Murray J. M.[5,6] suggests two models, in the first one includes a compartment of long-lived infected cells and in the second includes a pool of cells with full-length integrated HIV DNA.

What we suggest, is an extension of the basic model (Fig. 3) resulting in a simpler model to assess antiretroviral treatment efficacy. It may be less suited for long-term prediction. Consider instead of one compartment of infected cells, two, one with infected cells that can produce virus $\left(I_{1}\right)$ and one that because of Raltegravir is unable to produce virus $\left(I_{2}\right)$.

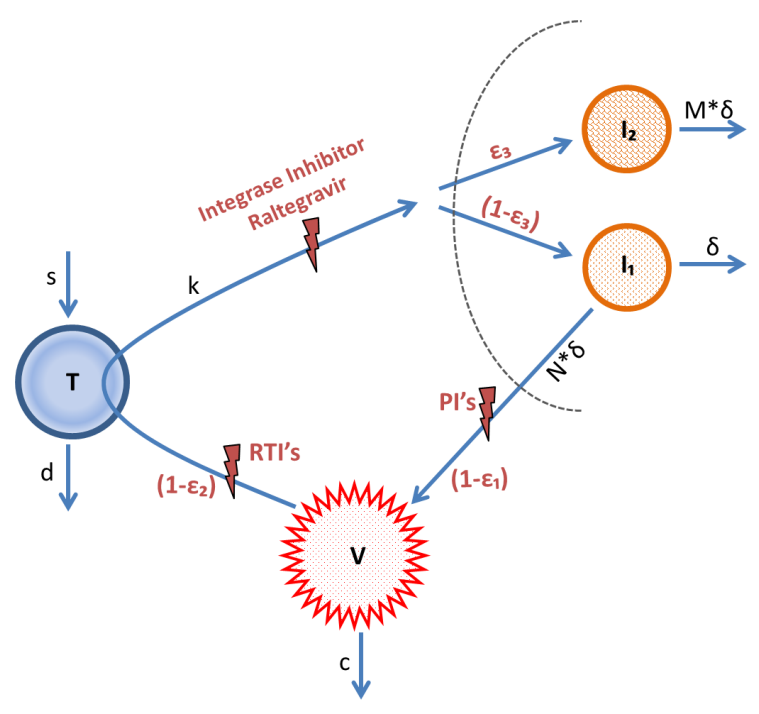

Fig. 3. Model of viral infection under combination treatment.

Thus our system of equations becomes:

$$
\begin{aligned}
& d T / d t=s-\left(1-\varepsilon_{2}\right) k V_{I} T-d T \\
& d I_{1} / d t=\left(1-\varepsilon_{2}\right)\left(1-\varepsilon_{3}\right) k V_{I} T-\delta I_{1} \\
& d I_{2} / d t=\left(1-\varepsilon_{2}\right) \varepsilon_{3} k V_{I} T-M \delta I_{2} \\
& d V_{I} / d t=\left(1-\varepsilon_{1}\right) N \delta I_{1}-c V_{I} \\
& d V_{N I} / d t=\varepsilon_{1} N \delta I_{1}-c V_{N I}
\end{aligned}
$$

where $M$ is the enhancement loss rate because of Raltegravir and $\varepsilon_{3}$ the efficacy of Raltegravir.

\section{FitTing EXAMPLE}

We consider an example of one patient of a recent study with patients infected with HIV-1 [13]. This patient was treated with 2 RTI's and Rltegravir.

In order to verify our model we fitted both measurements of plasma HIV RNA concentrations and CD4 T-cell counts with our model (Fig.5). All the calculations were performed with MATLAB. We solve our ODE system with ode-23s solver for stiff equations, we calculate the likelihood function and for the minimization process we used the fminsearch, which uses the Nelder-Mead algorithm.

We set the $M, N$ parameters fix and estimate $c, d$, $\delta, \varepsilon_{1}, \varepsilon_{2}, \varepsilon_{3}$ (Fig.4).

\begin{tabular}{|c|c|c|c|}
\hline \multicolumn{2}{|c|}{ Fix parameters } & \multicolumn{2}{|c|}{ Estimated parameters } \\
\hline $\mathrm{M}$ & 4 & $\mathrm{c}$ & $16.438 \mathrm{day}^{-1}$ \\
\hline $\mathrm{N}$ & 2000 & $\mathrm{~d}$ & $1.286 \mathrm{day}^{-1}$ \\
\hline \multirow{2}{*}{} & $\delta$ & $1.156 \mathrm{day}^{-1}$ \\
\cline { 2 - 4 } & $\varepsilon=1-\left(1-\varepsilon_{2}\right)\left(1-\varepsilon_{3}\right)$ & $0.919 \mathrm{day}^{-1}$ \\
\hline
\end{tabular}

Fig. 4. Fix and estimated parameters for our patient. Note that we have calculated $\mathrm{s}$ and $\mathrm{k}$ as: $\mathrm{s}=449.592$ cells $/$ day, $\mathrm{k}=2.52 \times 10^{-4}$

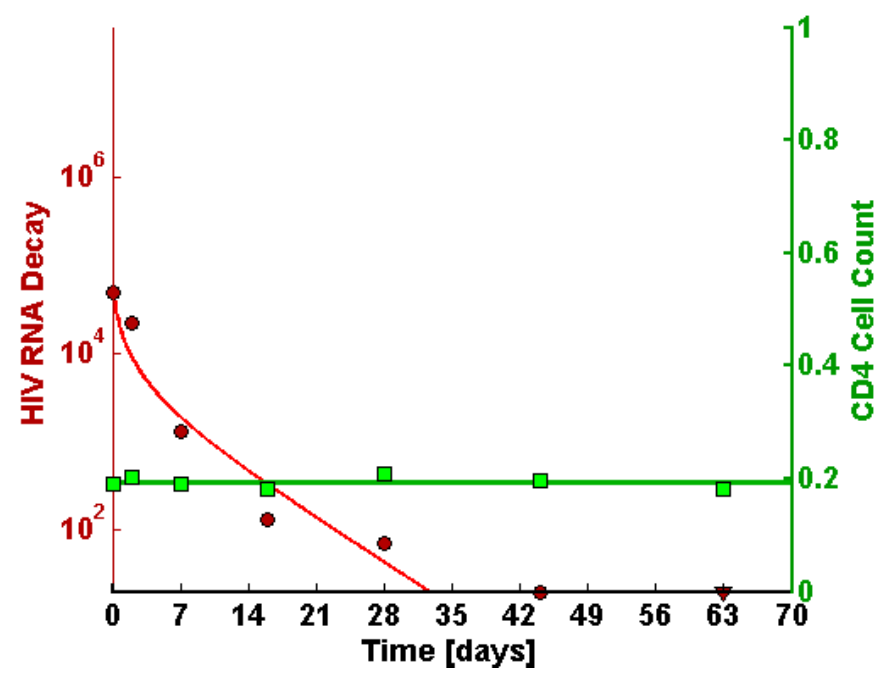

Fig. 5. Patient treated with Raltegravir and 2 RTI's

\section{EXTENDED Model}

As it is now also possible to quantify unintegrated DNA [13], we decided to extend our model. We know that the unintegrated DNA enter the nucleus, either integrates with the host DNA or binds with itself and 
forms 1-LTR (Long Terminal Repeat) and 2-LTR circles (Fig.6) $[2,12]$. Thus we introduce a new compartment $\left(I_{0}\right)$ of unintegrated DNA (Fig.7).

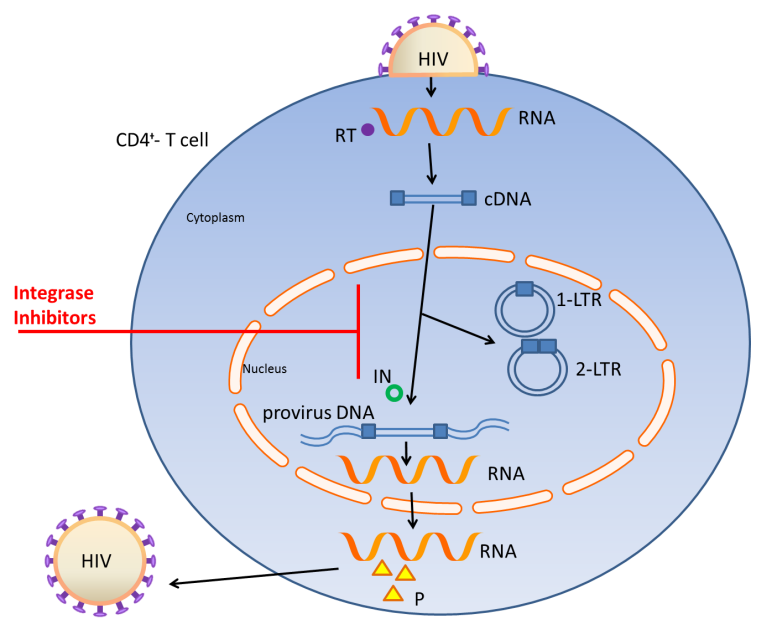

Fig. 6. Replication cycle-LTR circles.

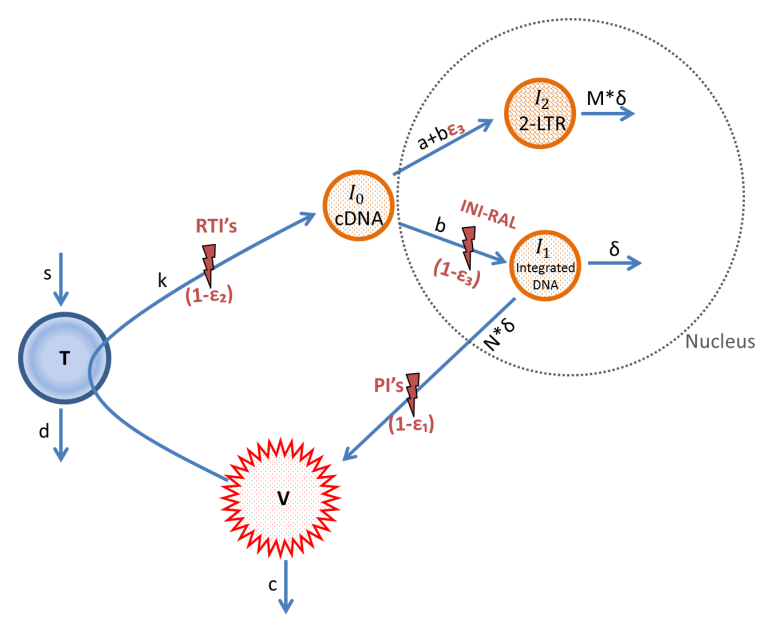

Fig. 7. Extended model of viral infection under combination treatment.

The ODE system that describes our model (Fig.7) is

$$
\begin{aligned}
& d T / d t=s-\left(1-\varepsilon_{2}\right) k T V_{I}-d T \\
& d I_{0} / d t=\left(1-\varepsilon_{2}\right) k T V_{I}-(a+b) I_{0} \\
& d I_{1} / d t=b\left(1-\varepsilon_{3}\right) I_{0}-\delta I_{1} \\
& d I_{2} / d t=\left(a+b \varepsilon_{3}\right) I_{0}-M \delta I_{2} \\
& d V_{I} / d t=\left(1-\varepsilon_{1}\right) N \delta I_{1}-c V_{I} \\
& d V_{N I} / d t=\varepsilon_{1} N \delta I_{1}-c V_{N I}
\end{aligned}
$$

where

- $a$ is the production rate of 2-LTR circles

- $b$ is the production rate of integrated DNA (before treatment $b>a$ )
- $I_{0}$ is the compartment of unintegrated DNA (cDNA)

- $I_{1}$ is the compartment of the 2-LTR circles

- $I_{2}$ is the compartment of integrated DNA

Fitting results of clinical data will be described in an upcoming clinical paper. Preliminary results on nonfinal data are promising.

\section{Conclusions}

Mathematical modelling of HIV-1 dynamics can be a really useful tool for the treatment of HIV-1 as they allow an efficient and early assessment of treatment efficacy. We can focus on viral clearance and CD4 Tcell death rates in which the medical and modelling community show interest, as well as the treatment efficacy parameters. Therefore we can also compare different treatment groups in order to see which treatment may be preferable. Overall, our model is relatively concise but may be less suited for longterm predictions. Previous models $[5,6,10,11]$ include a compartment of latently infected cells which makes them more complex and estimating treatment efficacy will be more difficult. Nevertheless, such models are better for fitting and predicting long-term clinical response. Overall we believe that the proposed model can be a tool to analyze data from clinical trials.

\section{REFERENCES}

[1] S. Bonhoffer, R.M. May, G.M. Shaw, M.A. Nowak, Virus dynamics and drug therapy, Proc. Natl. Acad. Sci. USA 49 6971-6976, 1997. http://dx.doi.org/10.1073/pnas.94.13.6971

[2] M. J. Buzon et al., HIV-1 replication and immune dynamics are affected by raltegravir intensification of HAART-suppressed subjects, Nature Medicine 162010.

[3] E. De Clercq, Strategies in the design of antiviral drugs, Nature Reviews Drug Discovery 1 13-25, 2002. http://dx.doi.org/10.1038/nrd703

[4] C. Hoffmann, J. K. Rockstroh, HIV 2011, Medizin Fokus Verlag, 2011. http://hivbook.com/

[5] J.M. Murray, S. Emery, A.D. Kelleher, M. Law, J. Chen, D.J Hazuda, B-Y T. Nguyen, H. Teppler and D.A. Cooper, Antiretroviral therapy with the integrase inhibitor raltegravir alters decay kinetics of HIV, significantly reducing the second phase, AIDS 21 2315-2321, 2007. http://dx.doi.org/10.1097/QAD.0b013e3282f12377

[6] J.M. Murray, HIV dynamics and integrase inhibitors, Antiviral chemistry and chemotherapy 19 157-164, 2002.

[7] M. A. Nowak and R. M. May, Virus Dynamics-Mathematical principles of immunology and virology, Oxford University Press, 2000.

[8] A. S. Perelson, P. W. Nelson, Mathematical Analysis of HIV-1 Dynamics in Vivo, SIAM Review 41:1 3-44, 1999. 
D. Bon et al., Viral Dynamic Model of Antiretroviral Therapy...

[9] A. S. Perelson, Modelling viral and immune system dynamics, Nature Reviews Immunology 2 28-36 January 2002. http://dx.doi.org/10.1038/nri700

[10] A. R. Sedaghat, J.B. Dinoso, L. Shen, C.O. Wilke, R.F. Siliciano,C.O. Wilke, Decay dynamics of HIV-1 depend on the inhibited stages of the viral life cycle, PNAS vol. 105, no. 12 4832-4837, 2008.

http://dx.doi.org/10.1073/pnas.0711372105

[11] A. R. Sedaghat, R.F. Siliciano, C.O. Wilke, Constraints on the dominant mechanism for HIV viral dynamics in patients on raltegravir, Antiviral Therapy 14 263-271, 2009.

[12] R. D. Sloan, M.A. Wainberg, The role of unintegrated DNA in HIV infection, Retrovirology 8:52 2011. http://dx.doi.org/10.1186/1742-4690-8-52
[13] C. Stephan, H.M. Baldauf, A. Haberl, M. Bickel, E. Herrmann, A. Berger, M. Stuermer, C. Goffinet, L. Kaderali, O. Keppler, Impact of Raltegravir on HIV-1 RNA and DNA Species Following Initiation of Antiretroviral Therapy CROI 2012, 19th Conference on Retroviruses and Opportunistic Infections.

[14] National Institute of Health-National Institute of Allergy and Infectious Diseases, HIV Replication Cycle Steps in the HIV Replication Cycle, 2012. http://www.niaid.nih.gov/topics/ HIVAIDS/Understanding/Biology/pages/hivreplicationcycle. aspx

[15] World Health Organization-UNAIDS, Global summary of the HIVIAIDS epidemic, 2010.

http://www.who.int/hiv/data/2011_epi_core_en.png 